\title{
Coulisses
}

Revue de théâtre

14 | Printemps 1996

Varia

\section{Les Règles du savoir-vivre dans la société moderne de Jean-Luc Lagarce}

Gisèle G. Holtzer

\section{OpenEdition}

1 Journals

Édition électronique

URL : https://journals.openedition.org/coulisses/4657

DOI : $10.4000 /$ coulisses.4657

ISSN : 2546-9460

Éditeur

Presses universitaires de Franche-Comté

Édition imprimée

Date de publication : 1 mai 1996

Pagination : 44-48

ISSN : 1150-594X

\section{Référence électronique}

Gisèle G. Holtzer, «Les Règles du savoir-vivre dans la société moderne de Jean-Luc Lagarce », Coulisses [En ligne], 14 | Printemps 1996, mis en ligne le 20 mars 2019, consulté le 15 février 2023. URL : http:// journals.openedition.org/coulisses/4657 ; DOI : https://doi.org/10.4000/coulisses.4657

Ce document a été généré automatiquement le 29 septembre 2020.

Tous droits réservés 


\title{
Les Règles du savoir-vivre dans la société moderne de Jean-Luc Lagarce
}

\author{
Gisèle G. Holtzer
}

La pièce est une coproduction du Théâtre de la Roulotte et du Théâtre Granit. Coaccueillie avec l'Espace, elle a été représentée au Nouveau Théâtre de Besançon du 23 au 27 avril 1996, dans une mise en scène de Jean-Luc Lagarce. Avant d'être jouée à Besançon, la pièce a eu 38 représentations. Créée en 1994 à Belfort, au Théâtre Granit (4 représentations), elle a été notamment à l'affiche du Théâtre de l'Athénée à Paris du 8 janvier au 10 février 1996 (30 représentations).

\section{Genèse de la pièce}

$1 \quad$ Les Règles du savoir-vivre dans la société moderne, c'est au départ un texte signé Jean-Luc Lagarce $^{1}$. Le thème (paradoxe apparent chez un auteur-metteur en scène dont le théâtre est perçu comme éloigné de préoccupations sociologiques et politiques) se place dans la continuité de questions sociales déjà explorées dans Les Serviteurs (1981), pièce qui parle des rapports de classe, et dans l'adaptation de J. Swift Instructions aux domestiques (1986). Dans cette dernière pièce, l'action se passe dans le sous-sol d'une maison de maîtres, une échelle servant de trait d'union entre "l'enfer » et les étages nobles, lieu de vie des maîtres dont « ceux d'en bas » perçoivent l'existence à travers les bruits du quotidien. «Le thème des domestiques avait toujours passionné Jean-Luc, explique Mireille Herbstmeyer, et il s'était dit qu'un jour il ferait un spectacle sur l'étage au-dessus $»^{2}$. C'est bien de la société «d'en haut » qu'il est question dans les Règles, société mondaine dont l'ordre repose sur l'observation stricte du code (rituels, conventions), garant de son pouvoir et de sa reproduction.

2 A l'origine, le texte est une commande de Henri Taquet du Théâtre Granit qui souhaitait une pièce pouvant être jouée dans le cadre de la formule «théâtre en appartement ». Cette contrainte explique le choix d'un spectacle à personnage unique. Jean-Luc Lagarce, auteur du texte, en a assuré la mise en scène - ce qui n'était pas prévu au départ - sur le grand plateau du Théâtre Granit et dans une salle de 600 
places, refusant, au nom du cérémoniel et du rituel théâtral (le théâtre a aussi ses codes), l'espace étroit de l'appartement.

\section{Pouvoir et références temporelles}

Les Règles (texte et représentation) sont construites sur un jeu complexe de références temporelles, confrontation des temps et des mondes représentés. En fait, le texte de Lagarce s'inspire d'un ouvrage écrit par la baronne Staffe, ouvrage paru en 1889 sous le titre Les Règles du savoir-vivre dans la société moderne. Dans la publication du texte de Jean-Luc Lagarce aucune mention n'est faite ni au texte source ni à la baronne Staffe. En inscrivant sa propre production sous le titre original, Jean-Luc Lagarce brouille (volontairement ?) les repères. Il exploite sur le plan de renonciation l'ambiguïté de la référence au temps du monde raconté. L'expression "société moderne» ne prend en effet sens que par rapport à un repère précis (la société moderne de la baronne Staffe n'est pas celle des années 90). Aussi le lecteur " naïf » peut-il être amené à penser qu'il sera question dans le texte de la société contemporaine, celle de son propre temps alors que le texte écrit en 1994 énonce en fait les règles en usage à la fin du XIX ${ }^{\text {ème }}$ siècle, parle donc apparemment d'une société dépassée, d'un monde révolu. Le caractère étrange, désuet, des rituels évoqués ("ça » ne se passe plus "comme ça» dans la société actuelle) fait que le lecteur recadre assez rapidement le temps du monde représenté. Mais le brouillage des temps pousse à l'interrogation. L'ordre de ce monde - expliqué, énoncé, commenté dans le détail de règles gouvernant l'existence entière de la naissance à la mort - a-t-il disparu? Quel est aujourd'hui le code secret de sa puissance?

4 La mise en scène joue sur un autre décalage temporel signifié par une sémiotique du mobilier et du vêtement. Le petit canapé, genre méridienne, ne correspond pas à un meuble façon XIX ${ }^{\text {ème }}$ siècle ; la robe de soie blanche de «la Dame » évoque une tenue de bal des années 50. Mireille Herbstmeyer éclaire cette « mise en abîme » des temps :

on a changé l'époque pour que ce soit aujourd'hui mais avec un décalage : c'est aujourd'hui, mais c'est suranné. La Dame est un peu dans la naphtaline. Elle est habillée à l'époque moderne sans que ce soit tout à fait aujourd'hui ; elle parle de règles qui sont encore plus anciennes mais ce monde n'est pas complètement révolu. Par exemple la cérémonie de l'argenterie se passe encore dans certaines familles.

5 Dans le texte, des indices ténus ancrent le texte dans le monde contemporain, créant une sorte d'anachronisme par irruption brusque du «moderne» dans l'«ancien». Ainsi, quand la mort entre dans une maison, « on baisse la radio » (p. 55); deux mois avant la fin du deuil, « on sifflote dans son bain » (p. 58). 


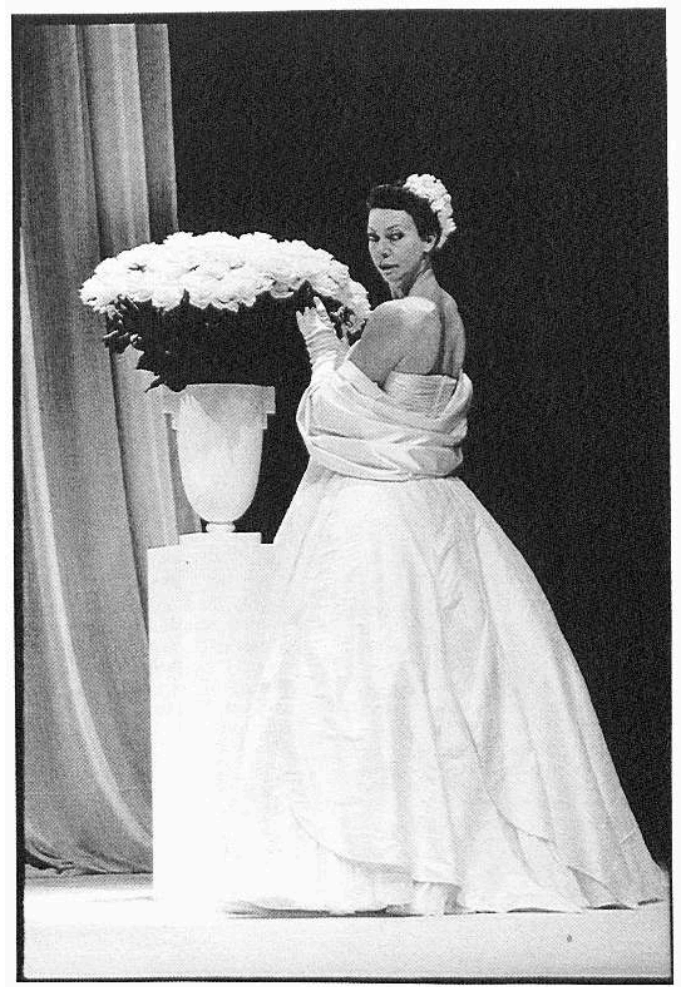

Photo Brigitte Enguerrand

6 Le regard contemporain projeté sur le monde d'autrefois (choc de deux mondes) peut susciter la curiosité amusée du lecteur/spectateur ethnographe intéressé par les mœurs et les usages d'une société en partie disparue; l'étonnement souriant, un tantinet sarcastique, du " moderne » contemplant le ridicule des archaïsmes sociaux; il peut aussi être empreint d'une sorte de nostalgie diffuse, regret d'un monde rassurant doté de repères forts où chacun sait comment se comporter « en toutes circonstances » et sait que l'autre suivra les mêmes règles; le regard contemporain peut aussi être animé du sentiment joyeux de la liberté « moderne » contre le carcan des normes et des règles sociales du passé. Ce serait oublier que le pouvoir social s'exprime au présent, dans l'implicite de règles et de codes qui excluent les uns, réaffirment la place des autres, la force du pouvoir étant le non dit, le secret, le non partagé qui laisse et maintient à distance le non initié3.

7 Par les décalages sur le temps, passé et présent s'interpénètrent, permanence de l'ordre perpétué du monde ("Ainsi que cela n'en finit jamais de se passer ») que le masque de rites poussiéreux ne doit pas nous empêcher de voir.

\section{Un texte à deux voix}

8 Dans la logique d'un texte qui s'inspire d'un livre source, deux voix se superposent, se juxtaposent, s'entremêlent. La voix de la baronne Staffe, c'est la voix de celle qui sait, voix pédagogique, assertive, qui avec assurance énonce les règles, explicite le code coutumier qui sous-tend le «bon » fonctionnement de la société dans toutes les étapes de la vie (naissance, fiançailles, mariage, deuil). Force du texte prescriptif, le style 
impersonnel domine, celui du texte sans auteur, où le sujet disparaît derrière la formulation générale de la " loi » (« il faut», « on doit»). La voix de Jean-Luc Lagarce subvertit ce matériau initial, par le commentaire, la prise de position, l'accentuation du ton « réglementaire »... Jean- Luc Lagarce, c'est la voix de la dérision, de l'ironie féroce, du cynisme (et du pessimisme) élégant.

On célèbre les noces d'argent après vingt-cinq ans d'heureuse union. L'union n'aurait-elle pas été heureuse, si elle dure vingt-cinq années, on la célèbre tout de même. On célèbre les noces d'argent après vingt-cinq années d'union. D'une manière plus générale, et pour résumer, en quelques circonstances que ce soit, si vingt-cinq années sont passées, on célèbre (p. 50).

(...)

Célébrons donc les noces d'argent, c'est un spectacle réconfortant (p. 51).

9 Tout cela est écrit avec de multiples points d'ironie, dit d'une voix ferme et alerte, visage lisse, image même de la conviction.

La répétition est un procédé d'écriture fréquemment présent dans le texte ; répétitions ternaires du devoir faire et du pouvoir faire :

Au cas où (...) vous seriez sollicité d'être parrain, pour être parrain, et dans le cas où vous souhaiteriez décliner cette offre (...), vous pouvez, pourriez, vous pouvez répondre, par exemple, c'est un moyen habile, vous pourriez répondre qu'au moment de la cérémonie, vous devez, devrez, vous devez accomplir un voyage (p. 14) ;

11 répétitions-reprises d'expressions, de formulations dont le retour un peu mécanique produit un effet de comique sous-tendu par la dérision : "répugnance et rien d'autre » (p. 13), « car ennuis et rien d'autre » (p. 14), « car disparu et rien d'autre» (p. 55), « car souvenir et rien d'autre» (p.5); ainsi en est-il des variations sur le «possible» et l'« envisageable », de la parole du poète Victor Hugo, etc. Ce discours de la répétition est le reflet dans l'écriture du caractère cyclique de la vie dont les actes sans cesse se reproduisent, leitmotiv qui ponctue la pièce : «ainsi que cela continue» (p. 12), « ainsi que toujours cela continue» (p.18), «toujours ainsi que cela continue» (p. 45), « et toujours ainsi que cela continue et recommence» (p.58), "Ainsi que cela n'en finit jamais de se passer » (p. 59), phrase qui clôt le texte.

Le travail d'écriture de Jean-Luc Lagarce est également sensible dans la manière dont il combine impersonnel/personnel, objectivité / subjectivité. La Dame s'exprime rarement en «je » et, lorsque c'est le cas, on constate une curieuse omission du « je » dans sa parole, effacement du moi derrière le code : « Mais si on veut bien y réfléchir, et suis là pour ça (...), ne saurais dire autrement, et explique pourquoi » (p. 12). A d'autres moments, le «je » est énoncé, mais dans des propos peu impliquants, orientés sur l'énonciation: «je n'insiste pas» (p.32), «je ne m'étends pas» (p.32), «je recommence » (p.16). Une seule et unique fois, le «je » trouve sa pleine expression, comme marque de la subjectivité - moment fugace de trouble du moi ; la Dame évoque le rituel des noces d'argent, «belle et grande fête de famille» et dit ceci : «C'est aussi une fête joyeuse, je pense ça, et on lui donne tout l'éclat possible » (p. 51).

\section{Mireille Herbstmeyer explique le personnage :}

Il y a un moment dans la pièce où la Dame dit «je pense ça »; partout ailleurs, elle ne dit pas ce qu'elle pense. Ici, c'est le moment dramatique du spectacle; on comprend qu'elle n'a rien vécu. Elle n'aura pas droit aux noces d'argent; c'est l'écroulement de son monde. 
La construction du personnage de la Dame prend sens rapportée au personnage réel de la baronne Staffe. De condition modeste, celle-ci ne s'est jamais mariée, a toujours vécu en marge de la société dont elle décrit les règles. A quarante-cinq ans, elle se mit à écrire son livre sur le savoir-vivre, travail d'écriture où, avec des mots, elle recrée l'univers où elle n'a pas eu sa place. C'est cet aspect de vie non accomplie, de faille secrète, qui a intéressé Jean-Luc Lagarce. « Cette femme qui apparemment n'a rien vécu s'est créé une espèce d'univers qui se rapproche du romanesque. Comme elle a toujours vécu par procuration, elle se retranche derrière quelque chose de très rigide, le code, ne laissant pas passer ses sentiments personnels ». La dimension dramatique, qui affleure de manière discrète, fugitifs instants de déséquilibre, est un fil ténu qui transparaît sous la tonalité majeure faite d'ironie et de distance ; c'est elle qui confère au personnage raide, impérieux, sa substance d'humanité.

\section{La représentation}

15 Sur la scène, tout est blanc, le décor et la toilette de la Dame : le canapé, la petite table, la boîte contenant l'argenterie; à gauche, un piédestal blanc supporte un vase blanc rempli de roses blanches. Blanc également le vélum au fond du salon, qui coupe la scène en deux espaces, l'espace du salon (devant le vélum), espace mondain où on se montre, où on reçoit, où on joue son rôle social; espace réservé (derrière le vélum), sorte de boudoir privé, lieu secret où l'on se retire (rarement) à l'abri des regards. Le blanc, couleur froide et éthérée, est la couleur thème du monde représenté tant dans le texte que dans la pièce. La symbolique en est donnée sur le mode duel du sérieux et de l'ironie :

Nous insistons à dessein assez souvent sur la couleur blanche, mais c'est parce que c'est la couleur de la foi, de la pureté, de la fidélité, de la vie, de la joie et de tas d'autres choses encore qui m'échappent à cette heure mais que la couleur blanche représente bien, aussi (p. 52).

Mireille Herbstmeyer, robe et étole de soie, coque dans les cheveux - blanches - donne au personnage la densité corporelle de sa stature, de sa prestance, sculpturale. Elle est la Dame, solidement altière, élégante, belle, dans la plénitude de la quarantaine, dont on se dit que sans conteste elle appartient au monde dont elle nous parle. La voix est ferme, le regard direct ; c'est une femme de conviction(s), qui maitrise les choses de la vie, de sa vie. Musique légère, support de rêve (la part du romanesque), Elisabeth Schwartzkopf chante un air des Légendes de la forêt viennoise, monde heureux où l'on célèbre, où l'on fête. L'interprète des Règles parle de son personnage : « La chose la plus importante que Jean-Luc m'a dite c'est «le personnage est sûr de lui. Tout ce que la Dame dit, elle l'affirme ; il faut complètement supprimer les rondeurs. » Le personnage, superbement incarné par Mireille Herbstmeyer, ne connaît pas l'hésitation ni le doute.

Elle a un côté masculin, un côté un peu grenadier par moments; en elle rien de langoureux, de féminin. Mais par moments quelque chose transparaît et on se dit que si elle s'était laissée aller, si la société le lui avait permis, sa vie se serait peutêtre transformée. Pour ma part, je ne la trouve pas très sympathique, mais elle a des excuses. 


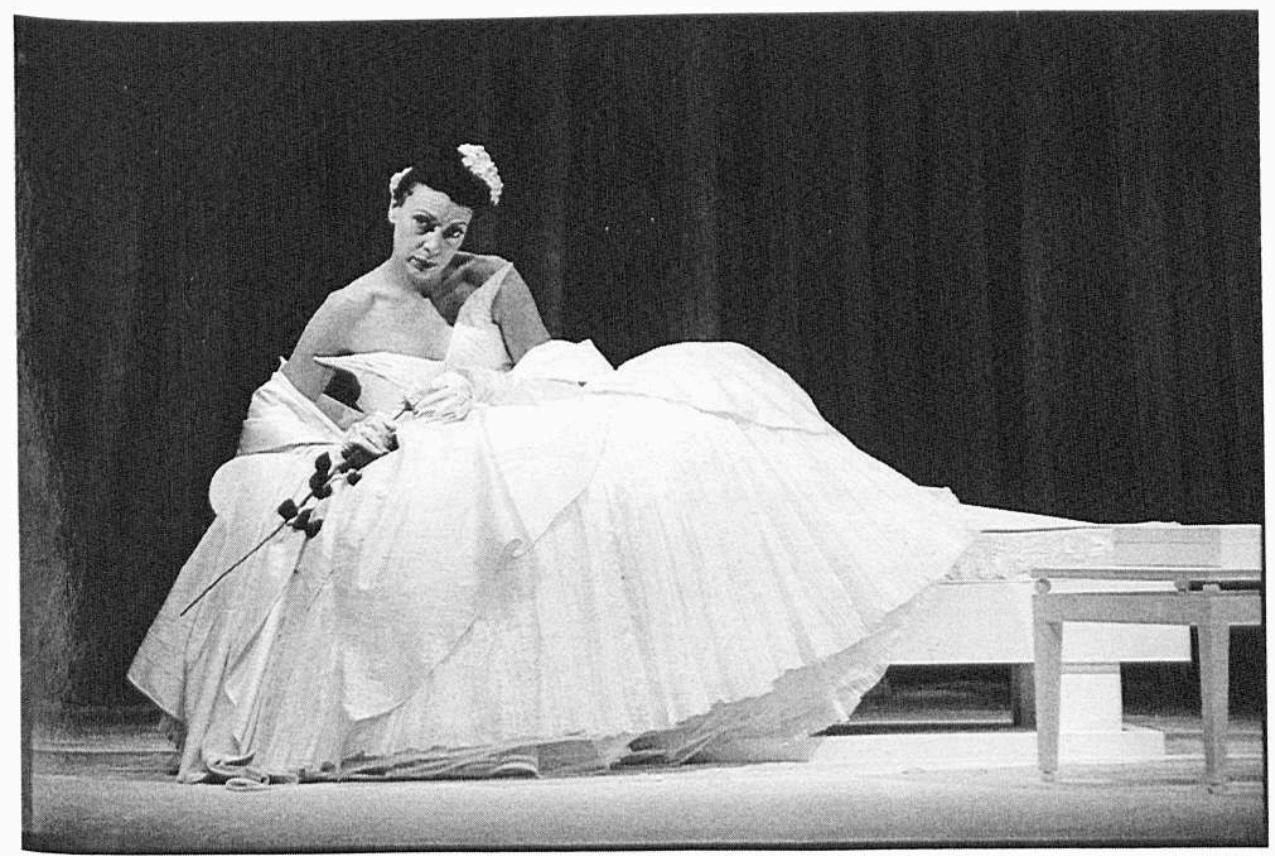

Photo Brigitte Enguerrand

17 Il y a des failles dans le personnage, failles suggérées par des discordances, entre discours et comportement par exemple. Avec son étole, elle astique les petites cuillers en argent qu'elle jette à terre tout en discourant, comme si de rien n'était, sur un monde ordonné et réglé; elle arrache d'une main «méchante » les pétales d'une rose qu'elle finit par décapiter furieusement; elle s'enroule comme frileusement dans son étole, en un geste de protection; contre quoi? Quelques rares moments, la parole se tait, le regard se perd, l'assurance se fissure, temps suspendu de l'émotion. C'est derrière le vélum que se passe le furtif moment dramatique du spectacle, celui des noces d'argent qu'elle ne vivra jamais. La Dame, placée de profil par rapport au public, laisse affleurer sa douleur intime - «je pense ça ». Par ces petites touches, discrètes et fugaces, se dessine le vide d'une destinée sublimée par un monde désiré recréé par le discours.

\section{Les Règles et l'espace de la représentation}

La pièce a été jouée à l'Athénée, dans une salle de 80 places alors qu'elle avait été créée dans une salle de 600 places. Mireille Herbstmeyer évoque l'effet de la dimension de l'espace de la représentation sur la signification de la pièce :

A l'Athénée, c'était comme si les gens étaient venus chez la Dame, dans son salon, écouter ses leçons de savoir-vivre. Il y avait un rapport de proximité, d'intensité, dû à la petitesse de la salle. Je m'adressais à chacun individuellement. Contrairement à ce qui se passe sur un grand plateau, à aucun moment le personnage n'avait une dimension de solitude. A l'Athénée, on a fait un travail de cisèlement sur le texte. Dans ce petit espace, le jeu est différent, ça joue sur des choses toutes petites, les déplacements sont limités. Le rapport à la salle était particulier: le public était placé en dessous de la scène et comme je suis grande, que j'ai une robe importante, on avait l'impression que je m'adressais à des nains - Sur le grand plateau du 
Théâtre Granit, ce qui ressort, c'est la dimension du vide ; la Dame est perdue dans un grand espace. Elle essaie de tenir le monde et en même temps, elle est dans le vide. Il y a un aspect plus tragique qu'on n'avait pas à l'Athénée. Le sens de la pièce passe mieux dans une grande salle.

\section{Comme une horloge comtoise}

19 Les Règles sont pour Mireille Herbstmeyer la première expérience de spectacle en interprète unique. Ici, le partenaire du personnage, c'est le public auquel elle adresse son monologue. L'absence d'appui pour la création d'un jeu dramatique, d'un rythme, est pour la comédienne une situation inédite, étrange. Interrogée sur la manière dont, seule sur scène, elle contrôle la durée de la représentation, elle avoue être régulière, le temps du spectacle ne variant que de une à deux minutes. "Jean-Luc me disait : "Tu es comme une horloge comtoise" ».

\section{NOTES}

1. Jean-Luc Lagarce, Les Règles du savoir-vivre dans la société moderne, Les Solitaires Intempestifs, 1996.

2. Les propos de Mireille Herbstmeyer, interprète des Règles, sont extraits d'une interview donnée le 22 avril 1996. La comédienne a fait partie de la distribution de la presque totalité des mises en scène de Jean-Luc Lagarce, à l'exception de Music-Hall, Phèdre et Lulu.

3. 3 II est intéressant de noter que le code est précisément explicité par la baronne Staffe, fausse baronne qui vécut en « dehors » du monde dont elle décrit les règles. 